\title{
Resultados del trasplante renal en portadores de riñones poliquísticos
}

\author{
JORGE VEGA ${ }^{1,2,3}$, DANIEL LIRA a , SIMÓN MEDEL ${ }^{\mathrm{a}}$, PABLO BETANCOUR ${ }^{2,3}$, \\ HELMUTH GOECKE ${ }^{2,3,4}$, ALEJANDRA CARRASCO ${ }^{2}$
}

'Servicio de Medicina Interna, Sección Nefrología, Hospital Dr. Gustavo Fricke, Viña del Mar.

${ }^{2}$ Servicio de Medicina Interna, Sección Nefrología, Hospital Naval A. Nef, Viña del Mar.

${ }^{3}$ Cátedra de Medicina del Hospital Naval A. Nef, Departamento de Medicina, Escuela de

Medicina, Universidad de Valparaíso, Valparaíso.

${ }^{4}$ Departamento de Nefrología, Hospital Clínico, Pontificia

Universidad Católica de Chile.

alumnos de la Escuela de Medicina, Universidad de Valparaíso.

Recibido el 26 de diciembre de 2011, aceptado el 18 de abril de 2012 .

Correspondencia a:

Dr. Jorge Vega Stieb, 5 Norte 1035, Viña del Mar.

Fono: 32-2974237

Fax: 32-2970050

E-mail: jvegastieb@gmail.

com

\section{Outcome of renal transplantation in patients with autosomal dominant polycystic kidney disease}

Background: Patients with autosomal dominant polycystic kidney disease $(A D P K D)$ have a better survival in chronic dialysis than patients with other etiologies of renal failure. It has been suggested that extra-renal manifestations of ADPKD may increase the odds of complications and mortality, if these patients are transplanted. Aim: To determine whether survival and complications in transplanted patients with $A D P K D$ are different from kidney graft recipients with other etiologies of renal failure. Subjects and Methods: Four hundred six patients with kidney transplantation were followed in three hospitals between 1976 and 2011 and 19 were carriers of ADPKD. The latter were matched by type of donor, gender, age and date of kidney transplant, with $38 \mathrm{graft}$ recipients with other etiologies of renal failure. Results: Graft and patient $1,5,10$ and 15 years survival were similar in both groups. Hospitalizations due to viral infections and sepsis were more common in patients with $A D P K D$. There were no differences in the rate of acute rejection, delayed graft function, cancer, gastrointestinal disorders and hospitalizations due to cardiovascular diseases. The frequency of graft loss due to death with a functioning kidney was similar between both groups. Conclusions: Patient and graft survival in transplanted patients with ADPKD were similar to patients with other etiologies of renal failure. The rate and type of complications were similar between groups with the exception of hospitalizations due to sepsis and viral infections, which were more common in ADPKD patients.

(Rev Med Chile 2012; 140: 990-998).

Key words: Kidney transplantation; Morbidity; Mortality; Polycystic kidney, autosomal dominant.

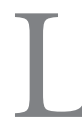

a enfermedad renal poliquística autosómica dominante (RPQ) es un desorden hereditario sistémico, que afecta principalmente a los riñones, frecuentemente al hígado y puede comprometer cerebro, páncreas, vasos arteriales y aparato digestivo ${ }^{1,2}$.

Su prevalencia es variable, 1 caso por cada 400-1.000 habitantes en Estados Unidos de Norteamérica, 1 por 2.459 habitantes en Gales y 1 por cada 4.033 habitantes en Japón ${ }^{3,4}$. En el mundo esta enfermedad afecta aproximadamente a 4-6 millones de personas ${ }^{5}$ y en Estados Unidos de Nor- teamérica a 300.000-600.000². Es la enfermedad genética que más frecuentemente origina una falla renal (IRC) con necesidad de diálisis ${ }^{1,6}$. El 50\% de los pacientes con RPQ llega a IRC entre la cuarta y sexta década de vida ${ }^{1,3,6}$.

La incidencia anual de IRC en Estados Unidos de Norteamérica secundaria a RPQ en hombres y mujeres es 8,7 y 6,9 casos por millón de habitantes y 5,6 y 4,0 en Japón ${ }^{4}$. En el mundo, de los pacientes en diálisis crónica (HDC), 2\% a 13\% son portadores de $\mathrm{RPQ}^{6-11}$. En Chile, 3,1\% de los pacientes en HDC son portadores de esta enfermedad ${ }^{12}$. 
La sobrevida de los pacientes portadores de RPQ en diálisis crónica es mejor a la de los dializados por IRC de otras etiologías ${ }^{10,11}$.

Dado que los enfermos con RPQ tienen con frecuencia alteraciones extrarenales como aneurismas aórticos o intracraneanos, valvulopatías cardiacas, quistes hepáticos o divertículos colónicos; podrían tener un mayor riesgo de sufrir complicaciones y morir a causa de ellas después de un trasplante renal $(\mathrm{TxR})^{11}$.

El objetivo de esta investigación fue conocer si los pacientes trasplantados renales portadores de RPQ tienen una sobrevida o complicaciones diferentes a los enfermos trasplantados por una falla renal de otra etiología.

\section{Sujetos y Métodos}

El estudio consideró 382 pacientes trasplantados entre 1968 y 2011 en los Hospitales Naval Almirante Nef, Dr. Gustavo Fricke y Clínica Reñaca y 24 que se controlaron regularmente en estos centros pero que fueron trasplantados en otros hospitales del país o del extranjero. Dieciséis enfermos recibieron un segundo trasplante y 2 un tercero. De los 406 pacientes, 19 (4,7\%) fueron portadores de RPQ y constituyen los casos incluidos en esta investigación. Cada uno de ellos fue pareado con 2 pacientes portadores de IRC de una causa diferente a RPQ (se excluyeron a los diabéticos) considerando su género, edad, tipo de donante (vivo o cadavérico), fecha de trasplante y hospital de la intervención o del seguimiento. Se eligió como controles a los dos pacientes trasplantados en la fecha más cercana al caso que cumplieran con todos los criterios señalados. Para el pareo de la edad se consideró que los controles tuvieran una diferencia menor de 10 años con el caso. No hubo pacientes con RPQ o controles que hayan sido excluidos por algún motivo, salvo el ser portadores de diabetes mellitus debido a que estos presentan mayor morbimortalidad.

En los 57 pacientes se registraron: datos demográficos, etiología de la enfermedad renal, tipo y características del donante, antigüedad del diagnóstico de la insuficiencia renal, tiempo de permanencia en diálisis, datos inmunológicos de donantes y receptores, fecha y lugar del trasplante, drogas inmunosupresoras utilizadas, evolución del TxR, complicaciones y el estado del paciente al final del seguimiento (1-12-2011).

\section{Estadística}

Para el análisis estadístico se utilizó frecuencias, porcentajes, promedios, desviación estándar, mediana y rango intercuartil según correspondiera; para determinar la distribución de las variables cuantitativas se graficaron los datos y se usó la prueba de normalidad de Shapiro-Wilk. El análisis inferencial bivariado se realizó mediante la prueba estadística de $\chi^{2}$ (chi cuadrado) con corrección de Yates para variables cualitativas, test exacto de Fisher cuando fue necesario y la prueba de MannWhitney para variables cuantitativas. El Odds Ratio fue la medida de riesgo con un intervalo de confianza de 95\%. Para las curvas de sobrevida se utilizó el método de Kaplan-Meier y para su comparación las pruebas de Log rank y Wilcoxon. Se consideró significativo un valor de $\mathrm{p}<0,05$. Se utilizó el programa estadístico Stata 10.0.

\section{Resultados}

Los pacientes portadores de RPQ y sus controles tuvieron donantes de edades similares y no difirieron en el número de incompatibilidades HLA, tasa de anticuerpos reactivos contra linfocitos de panel (PRA), número de trasplantes previos, duración de la insuficiencia renal entre el diagnóstico y el inicio de diálisis, tiempo de permanencia en diálisis ni en la prevalencia de infección crónica por los virus C y B de hepatitis. El donante del injerto en los 57 receptores fue mayoritariamente un sujeto en muerte cerebral (Tabla 1).

El esquema inmunosupresor inicial en la mayoría de los pacientes consistió en ciclosporina, azatioprina y prednisona. El uso de tacrolimus y micofenolato mofetil fue la excepción. Sin embargo, estas últimas drogas se fueron incorporando durante la evolución del TxR, así como rapamicina y everolimus. La inducción con anticuerpos antilinfocíticos o contra los receptores de IL-2 fue de baja ocurrencia, como lo fue también en la terapia de un rechazo agudo. Esta complicación tuvo una incidencia similar durante los primeros 5 años en ambos grupos. No hubo diferencias en las drogas inmunosupresoras empleadas durante la evolución del injerto (Tabla 2).

La función renal al momento del egreso hospitalario después de efectuado el trasplante, al año de evolución y en el último control fue similar entre los dos grupos (Tabla 3 ). 
Tabla 1. Características clínicas de 19 pacientes con riñones poliquísticos y de 38 controles trasplantados de riñón

\begin{tabular}{|c|c|c|c|c|c|}
\hline & & $\begin{array}{c}\text { Casos } \\
(n=19)\end{array}$ & $\begin{array}{l}\text { Controles } \\
(n=38)\end{array}$ & $\begin{array}{c}\text { Odds Ratio } \\
\text { (IC 95\%) }\end{array}$ & $\mathbf{p}$ \\
\hline Edad de los donantes (años) media & (DS) & $37,7 \quad(12,7)$ & $35,6(13,2)$ & & 0,61 \\
\hline Género de los donantes & n (\%) masculino & $(50,0)^{*}$ & $26 \quad(68,4)$ & $0,46(0,15-1.46)$ & 0,18 \\
\hline Donante fallecido & n (\%) & $(73,7)$ & $28 \quad(73,7)$ & $1,00(0,29-3,49)$ & $>0,99$ \\
\hline \multicolumn{2}{|c|}{ Edad de los receptores (años) media (DS) } & $47,9 \quad(5,4)$ & $45,1 \quad(8,3)$ & & 0,11 \\
\hline Género de los receptores & n (\%) masculino & $14 \quad(73,7)$ & $28 \quad(73,7)$ & $1,00(0,29-3,49)$ & $>0,99$ \\
\hline Primer trasplante renal & n (\%) & $(89,5)$ & $37 \quad(97,4)$ & $4,35(0,37-51,4)$ & 0,24 \\
\hline Segundo trasplante renal & n (\%) & $(10,5)$ & $1 \quad(2,6)$ & & \\
\hline PRA pre-trasplante mediana & $(\mathrm{RIC})$ & $(0,3)$ & $0 \quad(0,0)$ & & 0,36 \\
\hline PRA máximo mediana & $(\mathrm{RIC})$ & $3 \quad(0,25)$ & $3 \quad(0,5)$ & & 0,13 \\
\hline Incompatibilidades HLA A B media & (DS) & $2,4 \quad(1,0)$ & $2,0 \quad(0,8)$ & & 0,06 \\
\hline Incompatibilidades HLA DR media & (DS) & $0,6 \quad(0,8)$ & $0,6 \quad(0,5)$ & & 0,96 \\
\hline Intervalo entre Dg. IRC y HDC (mese & es) mediana (RIC) & $5(1,5,45,5)$ & $3,5(0,24)$ & & 0,52 \\
\hline Duración de HDC (meses) mediana & $(\mathrm{RIC})$ & $31,5(23,53)$ & $28,5(9,47)$ & & 0,65 \\
\hline Infección por virus $C$ de la hepatitis & n (\%) & $(18,8)$ & $6 \quad(18,8)$ & $1,00(0,21-4,65)$ & $>0,99$ \\
\hline Infección por virus B de la hepatitis & n (\%) & $(15,8)$ & $(2,6)$ & $6,94(0,67-71,9)$ & 0,10 \\
\hline \multicolumn{6}{|l|}{ Etiologías de la IRC: } \\
\hline - Riñones poliquísticos & n (\%) & $(100)$ & $(0,0)$ & & \\
\hline - Desconocida & n (\%) & $(0,0)$ & $19 \quad(50,0)$ & & \\
\hline - Glomerulonefritis crónica & n (\%) & $(0,0)$ & $15 \quad(39,5)$ & & \\
\hline - Pielonefritis crónica & n (\%) & $(0,0)$ & $(7,9)$ & & \\
\hline - Nefroesclerosis & n (\%) & $(0,0)$ & $(2,6)$ & & \\
\hline
\end{tabular}

PRA: anticuerpos reactivos contra linfocitos de panel; DM: diabetes mellitus; Dg: diagnóstico; RIC: rango intercuartil; *se desconoce el género de un donante.

La proporción de pacientes que tuvo una función renal retardada (requirió de diálisis después del TxR) fue similar entre los grupos. También lo fue la tasa de eventos cardiovasculares, neoplasias malignas, diabetes mellitus post TxR, daño hepático crónico, fracturas óseas, artritis gotosa y eritrocitosis. Los pacientes con RPQ tuvieron más frecuentemente hospitalizaciones por infecciones que los controles, sin embargo, la incidencia de infecciones urinarias no fue diferente (Tabla 4).

De los pacientes con RPQ que tuvieron una determinación previa al TxR de anticuerpos de tipo IgG para CMV, en 88,9\% de ellos estos fueron positivos. En los controles, la proporción de positivos fue $84,2 \%$. Todos los enfermos que sufrieron una infección por CMV fueron portadores de RPQ (Tabla 4).En la mayoría de ellos la serología para CMV se desconocía y no recibieron profilaxis antiviral (trasplantes efectuados antes de 1993).

El origen de las sepsis en los pacientes con RPQ fue urinario en $46,2 \%$, cutáneo en $23,1 \%$, desconocido en $15,4 \%$ y respiratorio o digestivo en los restantes. En los controles, el origen de las sepsis fue urinario en $50 \%$, digestivo en $37,5 \%$ y cutáneo en $12,5 \%$. Las sepsis fueron significativamente más frecuentes después de los 5 años en los pacientes con RPQ. Sólo en un enfermo fue necesario hacer una nefrectomía por quistes infectados recurrentes. No hubo infecciones de quistes hepáticos o complicación de los divertículos en ninguno de los pacientes con RPQ.

La sobrevida de los injertos (censurando a las muertes con riñón funcionante) a 1, 5, 10 y 15 años fue $100 \%, 100 \%, 91,7 \%$ y $91,7 \%$ en los portadores 
Trasplante renal en riñones poliquísticos - J. Vega et al

Tabla 2. Inmunosupresión utilizada en 19 trasplantados renales con riñones poliquísticos y sus 38 controles

\begin{tabular}{|c|c|c|c|c|c|}
\hline Características & & Casos & Controles & OR (IC 95\%) & $\mathbf{p}$ \\
\hline \multicolumn{6}{|l|}{ Inmunosupresión inicial } \\
\hline Azatioprina-prednisona & n (\%) & $4(21,1)$ & $10(26,3)$ & $0,75(0,20-2,80)$ & 0,66 \\
\hline Azatioprina-prednisona-CsA & $\mathrm{n}(\%)$ & $13(68,4)$ & $22(57,9)$ & $1,58(0,49-5,04)$ & 0,44 \\
\hline MMF-CsA- prednisona & n (\%) & $2(10,5)$ & $4(10,5)$ & $1,00(0,17-6,02)$ & $>0,99$ \\
\hline Otros esquemas & n (\%) & 0 & $2(5,2)$ & & \\
\hline Inducción con anticuerpos & n (\%) & $1(5,3)$ & $3(7,9)$ & $0,65(0,06-6,68)$ & 0,72 \\
\hline TAR durante el primer año del TxR & n (\%) & $7(36,8)$ & $19(50,0)$ & $0,58(0,19-1,80)$ & 0,35 \\
\hline MPDN gramos utilizados en año 1, mediana & $(\mathrm{RIC})$ & $0(0,1,5)$ & $0,5(0,3)$ & & 0,15 \\
\hline Uso de anticuerpos antilinfocíticos en TAR & n (\%) & $1(5,3)$ & $3(7,9)$ & & $>0,99$ \\
\hline \multicolumn{6}{|l|}{ Inmunosupresión al año de TxR } \\
\hline Azatioprina-prednisona & n (\%) & $4(21,0)$ & $6(17,6)$ & $1,18(0,30-4,67)$ & 0,81 \\
\hline Azatioprina-prednisona-CsA & n (\%) & $8(42,1)$ & $16(45,7)$ & $0,73(0,24-2,21)$ & 0,57 \\
\hline MMF-CsA- prednisona & n (\%) & $5(26,3)$ & $4(11,8)$ & $3,04(0,71-13,0)$ & 0,13 \\
\hline CsA- prednisona & n (\%) & $2(10,5)$ & $5(14,3)$ & $0,78(0,14-4,43)$ & 0,78 \\
\hline Otros esquemas & n (\%) & 0 & $3(8,6)$ & & \\
\hline TAR en años 2 al 5 post TxR & n (\%) & $2(10,5)$ & $5(15,2)$ & $0,70(0,12-4,03)$ & 0,69 \\
\hline Cambio en inmunosupresión & n (\%) & $12(63,2)$ & $18(47,4)$ & $1,90(0,62-5,89)$ & 0,26 \\
\hline \multicolumn{6}{|l|}{ Último esquema inmunosupresor utilizado } \\
\hline Azatioprina-prednisona & n (\%) & $2(12,5)$ & $9(27,3)$ & $0,38(0,07-2,02)$ & 0,26 \\
\hline Azatioprina-prednisona-CsA & n (\%) & $2(12,5)$ & $7(21,2)$ & $0,53(0,10-2,91)$ & 0,46 \\
\hline Ciclosporina-prednisona & n (\%) & $4(25,0)$ & $6(18,2)$ & $1,50(0,36-6,31)$ & 0,58 \\
\hline MMF- CsA-prednisona & n (\%) & $2(12,5)$ & $6(18,2)$ & $0,64(0,11-3,61)$ & 0,61 \\
\hline MMF-TAC-prednisona & n (\%) & $2(12,5)$ & $2(6,0)$ & $2,21(0,28-17,3)$ & 0,45 \\
\hline Otros esquemas & n (\%) & $4(24,8)$ & $2(6,0)$ & & \\
\hline
\end{tabular}

MMF: Micofenolato mofetil; CsA: Ciclosporina; TAC: Tacrolimus; TAR: Terapia antirechazo; TxR: Trasplante renal; MPDN: Metilprednisolona; RIC: rango intercuartil.

de RPQ y $100 \%, 90,5 \%, 82,8 \%$ y $75,9 \%$ en los controles, respectivamente. Estas diferencias no fueron significativas (Log rank test: 0,234; Wilcoxon: 0,204) (Figura 1).

La sobrevida de pacientes (censurando a quienes volvieron a HDC) a 1, 5, 10 y 15 años fue $100 \%, 89,2 \%, 57,6 \%$ y $32,0 \%$ en los portadores de ADPKD y $89,5 \%, 81,1 \%, 68,5 \%$ y $42,7 \%$ en los controles, respectivamente. Estas diferencias no fueron significativas (Log rank test: 0,786; Wilcoxon: 0,641) (Figura 2).

Las hospitalizaciones por eventos cardiovasculares y la incidencia de enfermedades digestivas o cáncer, no fueron estadísticamente diferentes entre los grupos (Tabla 5). La muerte con riñón funcionante fue la principal causa de pérdida de injertos $(76,5 \%)$ y su frecuencia fue similar entre los grupos. Las causas de muerte más frecuentes fueron las infecciones tanto en los pacientes con RPQ como en los controles. No hubo diferencias significativas entre los grupos en las causas de muerte (Tabla 5).

Al momento de corte en el seguimiento de esta serie, $52,6 \%$ de los portadores de RPQ y $34,2 \%$ de los controles estaban con su injerto funcionante (p NS). 
Tabla 3. Función renal durante la evolución del trasplante de 19 pacientes con RPQ y sus 38 controles

\begin{tabular}{|c|c|c|c|c|c|}
\hline Características & & Casos & Controles & OR (IC 95\%) & $\mathbf{p}$ \\
\hline Creatininemia al alta del TxR, mg/dl, mediana (RIC) & 1,46 & $6(1,0 ; 2,1)$ & $1,65(1,3 ; 2,1)$ & & 0,15 \\
\hline Creatininemia al año 1 del TxR,mg/dl, mediana (RIC) & 1,32 & $2(1,1 ; 1,7)$ & $1,38(1,1 ; 1,8)$ & & 0,56 \\
\hline eGFR al año $1\left(\mathrm{ml} / \mathrm{min} / 1,73 \mathrm{~m}^{2}\right)$ promedio (DS) & 59,1 & $(21,0)$ & $56,5 \quad(20,1)$ & & 0,64 \\
\hline Creatininemia al año 3 del TxR (mg/dl) promedio (DS) & 1,4 & $(0,4)$ & $1,5 \quad(0,5)$ & & 0,36 \\
\hline eGFR al año 3 (ml/min/1,73 m²) promedio (DS) & 56,1 & $(14,7)$ & $53,5 \quad(16,3)$ & & 0,55 \\
\hline Creatininemia al año 5 del TxR (mg/dl) promedio (DS) & 1,4 & $(0,5)$ & $1,5 \quad(0,5)$ & & 0,46 \\
\hline eGFR al año 5 (ml/min/1,73 m²) promedio (DS) & 55,5 & $(22,9)$ & $51,8 \quad(16,8)$ & & 0,69 \\
\hline Creatininemia al año 10 del TxR (mg/dl) promedio (DS) & 1,6 & $(0,6)$ & $1,8 \quad(0,5)$ & & 0,33 \\
\hline eGFR al año 10 (ml/min/1,73 m²) promedio (DS) & 53,1 & $(17,6)$ & $43,7 \quad(16,3)$ & & 0,32 \\
\hline $\begin{array}{l}\text { Última creatininemia ( } \mathrm{mg} / \mathrm{dl} \text { ) promedio (DS) en } \\
\text { controles ambulatorios en pacientes con MCRF }\end{array}$ & 1,7 & $(0,6)$ & $1,9 \quad(1,1)$ & & 0,09 \\
\hline $\begin{array}{l}\text { Última eGFR en controles ambulatorios en pacientes } \\
\text { con MCRF }\left(\mathrm{ml} / \mathrm{min} / 1,73 \mathrm{~m}^{2}\right) \text { promedio (DS) }\end{array}$ & 48,6 & $(17,9)$ & $34,6 \quad(14,6)$ & & 0,09 \\
\hline $\begin{array}{l}\text { Duración del TxR (meses), promedio (DS) en pacientes } \\
\text { con MCRF }\end{array}$ & 95,9 & $(50,9)$ & $110,4(89,5)$ & & 0,83 \\
\hline Edad al fallecimiento (años), promedio (DS) & 57,6 & $(5,7)$ & $57,2 \quad(10,4)$ & & 0,80 \\
\hline $\begin{array}{l}\text { Última creatinina ambulatoria }(\mathrm{mg} / \mathrm{dl}) \text { mediana }(\mathrm{RIC}) \text { en } \\
\text { pacientes con riñón funcionante a la fecha de corte }\end{array}$ & 1,2 & $(0,9 ; 1,4)$ & $1,5 \quad(1,4 ; 1,8)$ & & 0,06 \\
\hline $\begin{array}{l}\text { Última eGFR (ml/min/1,73 } \mathrm{m}^{2} \text { ) promedio (DS) en } \\
\text { pacientes con riñón funcionante a la fecha de corte }\end{array}$ & 61,0 & $(25,7)$ & $51,1 \quad(15,0)$ & & 0,21 \\
\hline $\begin{array}{l}\text { Duración del TxR (meses), promedio (DS) en pacientes } \\
\text { que regresaron a HDC }\end{array}$ & 90 & $(\mathrm{NE})$ & $63,2(48,8)$ & & 0,32 \\
\hline Pacientes con pérdida del injerto y regreso a HDC n (\%) & 1 & $(5,26)$ & $(15,79)$ & $0,29(0,01-2,80)$ & 0,41 \\
\hline Pacientes que murieron con riñón funcionante & 8 & $(42,11)$ & $(50,00)$ & $0,72(0,20-2,53)$ & 0,78 \\
\hline Pacientes con injerto funcionante a fecha de corte n (\%) & 10 & $(52,63)$ & $(34,21)$ & $2,14(0,60-7,60)$ & 0,25 \\
\hline
\end{tabular}

TxR: Trasplante renal; eGFR: Velocidad de filtración glomerular estimada con fórmula MDRD; med: mediana; RIC: rango intercuartil; MCRF: Muerte con riñón funcionante; HDC: Hemodiálisis crónica. N.E: No hay DS ya que sólo fue 1 caso.

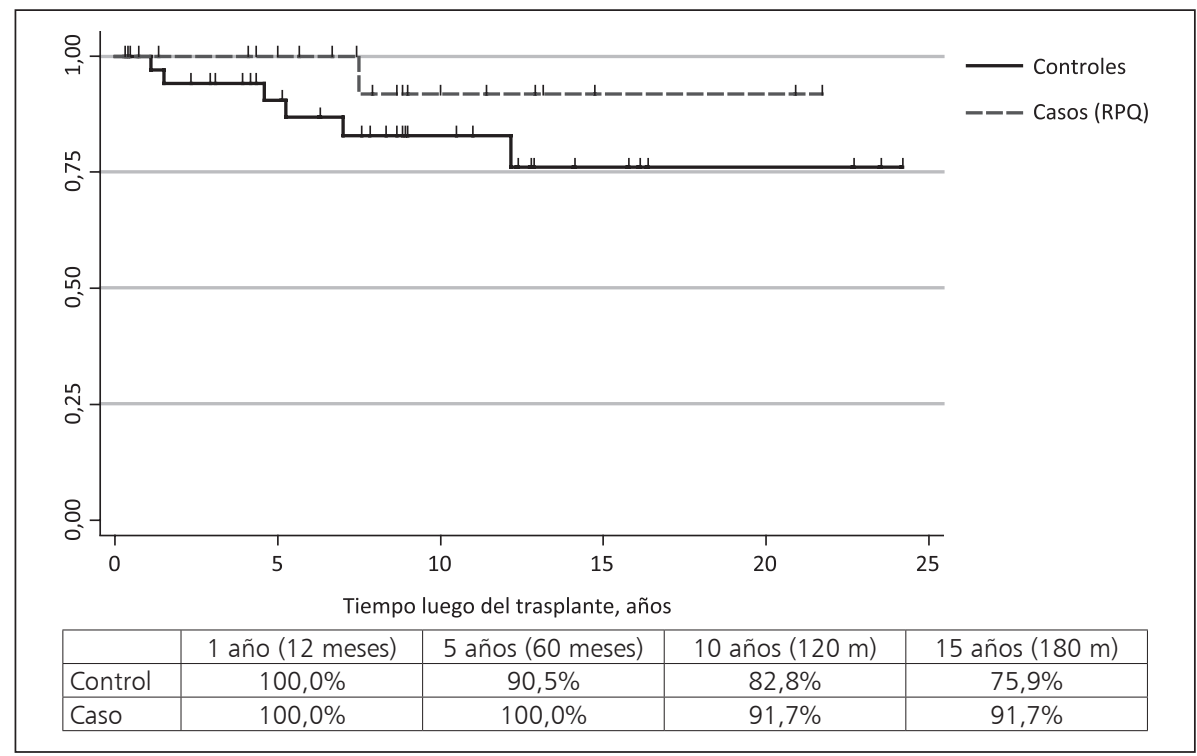

Figura 1. Sobrevida de injertos en 19 pacientes con RPQ y 38 controles trasplantados de riñón (se censuraron a los que fallecieron con un injerto funcionante). Log-rank test: 0,234. Wilcoxon: 0,204. 
Trasplante renal en riñones poliquísticos - J. Vega et al

Tabla 4. Complicaciones del trasplante renal en 19 pacientes con RPQ y sus 38 controles

\begin{tabular}{|c|c|c|c|c|c|}
\hline Complicaciones & & $\begin{array}{c}\text { Casos } \\
\%\end{array}$ & $\begin{array}{c}\text { Controles } \\
\%\end{array}$ & $\begin{array}{l}\text { Odds ratio } \\
\text { (IC 95\%) }\end{array}$ & $\mathbf{p}$ \\
\hline Función renal retardada & $(\%)$ & 21,0 & 26,3 & $0,75(0,15-3,2)$ & 0,66 \\
\hline Hospitalizaciones por infecciones en primeros 5 años & $(\%)$ & 57,9 & 21,1 & $5,16(1,55-17,1)$ & 0,007 \\
\hline Enfermedades cardiovasculares en primeros 5 años & $(\%)$ & 21,1 & 13,2 & $1,76(0,41-7,50)$ & 0,44 \\
\hline Neoplasias malignas en primeros 5 años & $(\%)$ & 10,5 & 5,3 & $2,12(0,27-16,3)$ & 0,47 \\
\hline Diabetes Mellitus post TxR & $(\%)$ & 26,3 & 13,2 & $2,36(0.59-9,45)$ & 0,23 \\
\hline Daño hepático crónico & $(\%)$ & 21,1 & 10,5 & $2,27(0.50-10,3)$ & 0,29 \\
\hline Alcoholismo & $(\%)$ & 15,8 & 5,3 & $3,38(0,51-22,2)$ & 0,21 \\
\hline Eritrocitosis post $T x R$ & $(\%)$ & 21,0 & 21,0 & $1,00(0,26-3,86)$ & $>0,99$ \\
\hline Infección por virus varicela-zoster & $(\%)$ & 31,6 & 23,7 & $1,49(0,44-5,05)$ & 0,52 \\
\hline Infección por citomegalovirus & $(\%)$ & 15,8 & 0 & & 0,03 \\
\hline Tuberculosis & $(\%)$ & 10,5 & 2,6 & $4,35(0,37-51,4)$ & 0,24 \\
\hline Infección urinaria en primeros 5 años & $(\%)$ & 26,3 & 39,5 & $0,57(0,13-2,08)$ & 0,39 \\
\hline$\geq 3$ infecciones urinarias en primeros 5 años & $(\%)$ & 21,0 & 5,3 & $4,80(0,59-56,6)$ & 0,09 \\
\hline Fracturas óseas & $(\%)$ & 15,8 & 5,3 & $3,38(0,51-22,2)$ & 0,21 \\
\hline Necrosis ósea avascular & $(\%)$ & 10,5 & 2,6 & $4,35(0,37-51,4)$ & 0,24 \\
\hline Gota aguda & $(\%)$ & 10,5 & 13,2 & $0,78(0,14-4,43)$ & 0,78 \\
\hline Hospitalizaciones por infecciones después del $5^{\circ}$ año & $(\%)$ & 78,6 & 34,8 & $6,88(1,48-32,0)$ & 0,01 \\
\hline Enfermedades cardiovasculares después del $5^{\circ}$ año & $(\%)$ & 7,1 & 26,1 & $0,22(0,02-2,04)$ & 0,18 \\
\hline Neoplasias malignas después del $5^{\circ}$ año & $(\%)$ & 28,6 & 29,1 & $1,13(0,26-5,01)$ & 0,87 \\
\hline Infección urinaria después del 5 año & $(\%)$ & 50,0 & 25,0 & $3,00(0,60-15,1)$ & 0,16 \\
\hline$\geq 3$ infecciones urinarias después del 5 año & $(\%)$ & 14,3 & 12,5 & $1,17(0,09-11,7)$ & 0,99 \\
\hline
\end{tabular}

TxR: Trasplante renal.

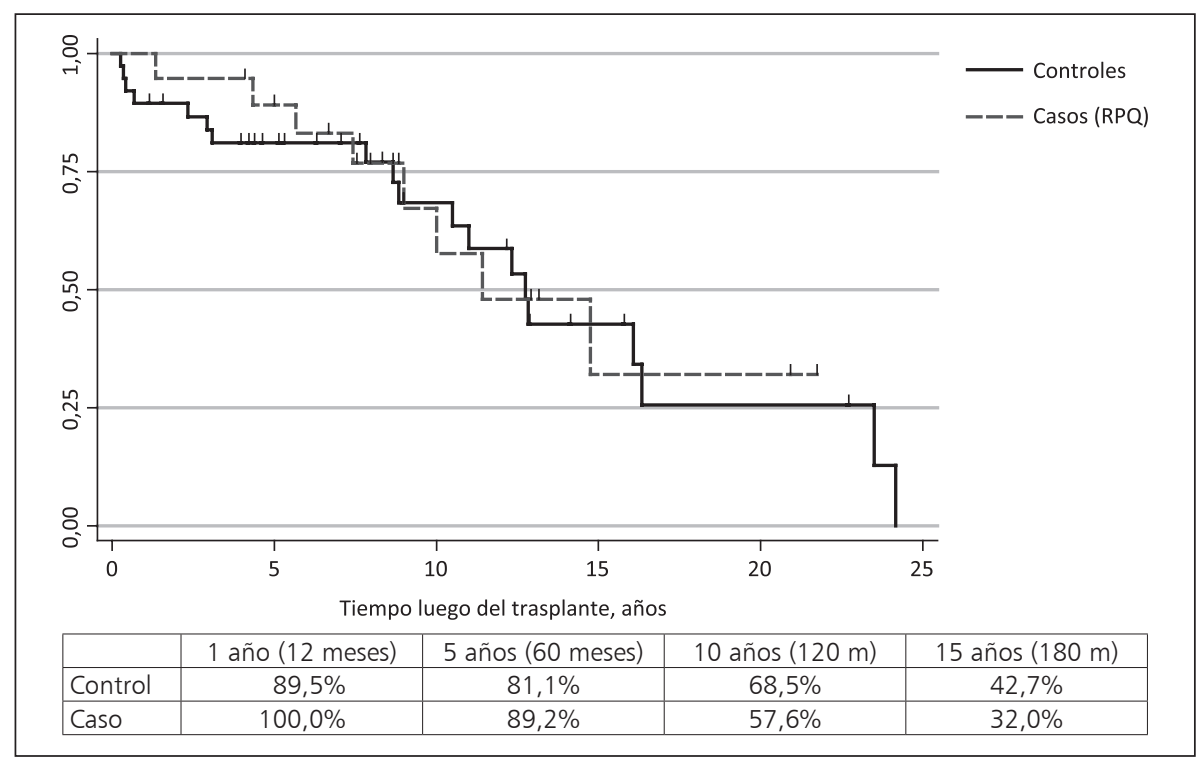

Figura 2. Sobrevida en 19 sujetos con RPQ y 38 controles trasplantados de riñón (se censuraron a los que regresaron a diálisis crónica. Logrank test: 0,786 . Wilcoxon: 0,641 . 
Tabla 5. Hospitalizaciones por eventos CV, incidencia de enfermedades digestivas y cánceres. Causas de muerte con riñón funcionante

\begin{tabular}{|c|c|c|c|}
\hline & RPQ & Controles & $\mathbf{p}$ \\
\hline Primeros 5 años & $\begin{array}{c}(n=19) \\
\%\end{array}$ & $\begin{array}{c}(n=38) \\
\%\end{array}$ & \\
\hline Enfermedades digestivas & 15,8 & 10,5 & 0,57 \\
\hline Enfermedades cardiovasculares & 21,0 & 10,5 & 0,28 \\
\hline Cáncer & 5,3 & 2,6 & 0,61 \\
\hline Año 6 en adelante & $\begin{array}{c}(n=15) \\
\%\end{array}$ & $\begin{array}{c}(n=24) \\
\%\end{array}$ & \\
\hline Enfermedades digestivas & 53,3 & 29,2 & 0,13 \\
\hline Enfermedades cardiovasculares & 6,7 & 29,2 & 0,09 \\
\hline Cáncer & 33,3 & 29,2 & 0,78 \\
\hline $\begin{array}{l}\text { Muerte con riñón funcionante } \\
\text { Causas }\end{array}$ & $(n=8 / 19) \quad 42,1 \%$ & $(n=18 / 38) \quad 47,4 \%$ & 0,71 \\
\hline Infecciones & 50,00 & 38,9 & 0,59 \\
\hline Enfermedades digestivas & 25,00 & 22,2 & 0,88 \\
\hline Enfermedades cardiovasculares & 0 & 27,8 & 0,09 \\
\hline Cáncer & 25,00 & 11,1 & 0,36 \\
\hline
\end{tabular}

CV: Cardiovasculares; TxR: Trasplante renal; RPQ: Riñones poliquísticos; HDC: Hemodiálisis crónica.

\section{Discusión}

En esta serie la prevalencia de RPQ como causa de IRC fue $4,7 \%$. Esta proporción ha variado entre $6,5 \%$ y $15 \%$ en diversas publicaciones ${ }^{6-8,11,13-15}$.

\section{Sobrevida de injertos}

En nuestra serie, la sobrevida de injertos a 1, 5,10 y 15 años en pacientes con RPQ no fue estadísticamente diferente a la de los controles. Estos resultados han sido encontrados también por otros autores ${ }^{2,8,13,16}$. En 1990 Fitzpatrick et al comunicaron los resultados de los TxR efectuados en Clínica Mayo a 54 pacientes con RPQ entre 1964-1987, los que fueron pareados con 107 pacientes con otras enfermedades renales. Encontraron que la sobrevida de injertos en ambos grupos fue similar a 1, 5 y 10 años de efectuado el $\mathrm{TxR}^{8}$. Florijn et al publicaron en 1994 una serie de $101 \mathrm{TxR}$ con RPQ, efectuados en Holanda entre 1967-1991, los que compararon con 692 controles con otras nefropatías. Encontraron que la sobrevida de los injertos en pacientes con RPQ no fue diferente a la de sus controles ${ }^{17}$. Hallazgos similares fueron publicados en 1997 por Hadimeri et al quienes en 114 pacientes con RPQ, trasplantados en Suecia entre 1985-1993, encontraron una sobrevida de injertos a 5 años de
$62 \%$ vs $61 \%$ en los controles ${ }^{13}$. El año 2002 Stiasny et al reportaron una serie de $90 \mathrm{TxR}$ con RPQ que fueron pareados con 88 pacientes injertados durante el mismo período. La sobrevida de injertos a 1 , 5 y 10 años fue $83 \%, 73 \%$ y $67 \%$, respectivamente, en los pacientes con RPQ y $84 \%, 70 \%$ y $51 \%$ en los controles, diferencias que no fueron significativas ${ }^{16}$.

Estos hallazgos no han sido universales. Algunos autores han encontrado mejor sobrevida de injertos en pacientes trasplantados con RPQ al compararlos con sujetos con otras etiologías de IRC $^{1,7}$. Johnson et al el año 2005 comunicaron los resultados de $187 \mathrm{TxR}$ efectuados en pacientes con RPQ en Irlanda, entre 1989-2002, los que fueron comparados con 1.080 sujetos con otras patologías renales. Encontraron que la sobrevida de los injertos a 1,5 y 10 años fue $88,2 \%, 79,1 \%$ y $54,3 \%$ en los pacientes con RPQ y $85,5 \%, 68,1 \%$ y $48,6 \%$ en los controles, respectivamente, diferencias que fueron significativas ${ }^{7}$. Las variables que influyeron en el pronóstico fueron la edad del receptor y del donante, tasa de PRA, género del donante y función retardada del injerto. El año 2011 Jacquet et al publicaron una serie de $543 \mathrm{TxR}$ en pacientes con RPQ, entre los años 1988-2007 en Francia. Estos fueron comparados con 4.779 con otras nefropatías. Encontraron que la sobrevida de injertos 
Trasplante renal en riñones poliquísticos - J. Vega et al

(censurando las muertes con riñón funcionante) a los 5, 10 y 15 años del TxR fue 90,4\%, 81,1\% y $76 \%$ en los pacientes con RPQ y $86,9 \%, 75,4 \%$ y $66 \%$, en los con otras nefropatías, respectivamente. Estas diferencias fueron significativas ${ }^{1}$.

\section{Complicaciones}

Los portadores de RPQ en IRC habitualmente ingresan a HDC y son trasplantados a una edad superior a la de los pacientes con otras nefropatías y tienen una mayor prevalencia de hipertensión arterial, hipertrofia ventricular izquierda, enfermedad diverticular del colon, valvulopatías cardiacas, aneurismas intracraneanos y aórticos, quistes hepáticos y pancreáticos ${ }^{2,6,9,6,18}$. Por ello se ha planteado que estos pacientes al ser sometidos a un TxR, tendrían un mayor riesgo de complicaciones y de muerte por causas cardiovasculares o infecciosas, principalmente infecciones de los quistes renales y hepáticos y complicaciones de los divertículos colónicos ${ }^{16}$. En los pacientes con RPQ se ha comunicado mayor frecuencia de adenomas renales por lo que se ha temido que puedan sufrir una mayor incidencia de carcinomas. Los enfermos con RPQ tienen frecuentemente resistencia a la insulina por lo que se ha postulado que el TxR, al requerir corticoides en su esquema terapéutico, aumentaría la prevalencia de diabetes mellitus post trasplante (DMPT) y que ella aumentaría el riesgo de sufrir infecciones urinarias ${ }^{9}$.

Algunas publicaciones han comunicado que no existen diferencias en la incidencia de complicaciones posteriores al TxR entre los pacientes con RPQ y sus controles ${ }^{6,10}$. Otras, como Fitzpatrick et al han comunicado una mayor frecuencia de complicaciones relacionadas con las manifestaciones extra-renales de la enfermedad como: disección aórtica, aneurismas intracraneales, insuficiencia valvular aórtica y quistes infectados; sin embargo, no observaron una mayor frecuencia de diverticulitis, perforación colónica ni cáncer renal ${ }^{8}$. Jacquet et al en su serie de pacientes con RPQ encontraron una mayor prevalencia de DMPT y de enfermedad tromboembólica, pero no observaron una mayor frecuencia de eventos cardiovasculares, accidentes cerebrovasculares, infecciones bacterianas ni cánceres renales ${ }^{1}$. Stiazny et al en 90 pacientes TxR con RPQ no encontraron una mayor frecuencia de cardiopatía coronaria, enfermedad tromboembólica, infecciones ni de neoplasias, respecto a los controles. Si encontraron en cambio, una mayor incidencia de infecciones urinarias ${ }^{16}$. Este hallazgo no fue encontrado en nuestra serie. Florijn et al en 101 pacientes con RPQ encontraron una mayor incidencia de infarto al miocardio y accidentes cerebrovasculares que en los controles, en los que usaron azatioprina. Ello no ocurrió en los que usaron ciclosporina ${ }^{17}$. En nuestra serie, en que más de 70\% de los pacientes utilizó ciclosporina, la incidencia de hospitalizaciones por complicaciones cardiovasculares no fue estadísticamente diferente entre los pacientes con RPQ y sus controles. Vicente Torres, en un artículo de revisión destacó que las complicaciones directamente relacionadas con la enfermedad son raras ${ }^{4}$, lo que también fue observado en nuestra serie.

\section{Sobrevida de pacientes}

En nuestra serie la sobrevida de pacientes a 5, 10 y 15 años no fue estadísticamente diferente en los pacientes con RPQ y sus controles. Perrone et al, seleccionó a todos los pacientes con RPQ TxR en Estados Unidos de Norteamérica $(\mathrm{n}=3.170)$ que iniciaron diálisis crónica entre 1990-1996 y los parearon con sujetos con otras etiologías de IRC. Encontraron que la sobrevida de los pacientes trasplantados con RPQ fue similar a la de los controles y que sus causas de muerte no fueron diferentes. No hubo influencia de las manifestaciones extrarenales de los RPQ en la mortalidad. La duración de la diálisis en el período previo al TxR no influyó sobre la mortalidad en ninguno de los 2 grupos ${ }^{11}$. Johnson et al, en 187 trasplantados con RPQ no encontraron diferencias en la sobrevida de pacientes respecto a los controles. La sobrevida a 1, 5 y 10 años fue $98 \%, 95,4 \%$ y $81,8 \%$ en los con RPQ y $96 \%$, $89 \%$ y $73,4 \%$ en los controles, respectivamente ${ }^{7}$. Jacquet et al encontraron que la sobrevida a 5,10 y 15 años en pacientes con RPQ fue similar a la de sus controles. Ella fue 93,4\%, $87,4 \%$ y $78,7 \%$ y $93,4 \%, 87,2 \%$ y $82,4 \%$, respectivamente ${ }^{1}$. Stiasny et al, en 90 TxR con RPQ no encontraron diferencias en la sobrevida de pacientes, sin embargo, observaron que la sobrevida de las mujeres a 10 años fue significativamente superior a la de los hombres. Esta diferencia de sobrevida por género no se encontró en los controles ${ }^{16}$. Florijn et al, en 101 pacientes con RPQ encontraron una sobrevida de pacientes similar a la de los controles, pero una mayor frecuencia de muerte con riñón funcionante por enfermedades cardiovasculares ${ }^{17}$. Brazda et al, en 104 injertos en pacientes con RPQ 
Trasplante renal en riñones poliquísticos - J. Vega et al

observaron una sobrevida a 5 años de $95 \%$, la cual no fue diferente a la de los controles, $90 \%$. En esa serie, los pacientes sometidos a nefrectomía de los riñones poliquísticos previo al TxR tuvieron una sobrevida mayor a 1 y 5 años $(100 \%)$ que los que no fueron nefrectomizados $(92 \%, 84 \%)^{14}$. En nuestros pacientes con RPQ ninguno fue nefrectomizado previo al TxR.

En suma, en nuestra serie de TxR portadores de RPQ la sobrevida de injertos y pacientes no fue estadísticamente diferente respecto a la de sus controles. Este hallazgo sugiere que los pacientes con $R P Q$, que habitualmente son significativamente mayores que los trasplantados con otras etiologías de falla renal, no se comportan en forma diferente, por lo que debieran ser incorporados a las listas de trasplante usuales y no necesariamente a las listas de donantes con criterio expandido. La frecuencia de complicaciones fue similar entre los grupos con excepción de una mayor frecuencia de hospitalizaciones por infecciones virales y septicemias en los portadores de RPQ, lo que nos permite proponer una vigilancia más activa al respecto en ellos. La muerte con un riñón funcionante fue la principal causa de pérdida de los injertos y no hubo diferencias en las causas de muerte entre ambos grupos. Las infecciones fueron la causa más frecuente de muerte con riñón funcionante. No hubo influencia de las manifestaciones extrarenales de los RPQ en la morbilidad ni mortalidad.

\section{Referencias}

1. Jacquet A, Pallet N, Kessler M, Hourmant M, Garrigue $\mathrm{V}$, Rostaing L, et al. Outcomes of renal transplantation in patients with autosomal dominant polycystic kidney disease: a nationwide longitudinal study. Transpl Int 2011; 24: 582-7.

2. Grantham JJ. Autosomal dominant polycystic kidney disease. Ann Transplant 2009; 14: 86-90.

3. Sulikowski T, Tejchman K, Zietek Z, Rózański J, Domański L, Kamiński M, et al. Experience with autosomal dominant polycystic kidney disease in patients before and after renal transplantation: a 7-year observation. Trasplantation Proceedings 2009; 41: 177-80.

4. Torres VE, Harris PC, Pirson Y. Autosomal dominant polycystic kidney disease. Lancet 2007; 369: 1287-301.

5. Amirzargar MA, Dadras F, Khoshjoo F, Yavangi M, Jafary $\mathrm{M}$, Bahar SH, et al. Non-Hodgkin's lymphoma in autosomal dominant polycystic kidney disease, 12 years after renal transplantation. Saudi J Kidney Dis Transplant
2007; 18: 419-21.

6. Roozbeh J, Razmkon AR, Jalaeian H, Raiss-Jalali GA, Behzadi S, Sagheb MM, et al. Outcome of kidney transplantation in patients with polycystic kidney disease: a single center study. Saudi J Kidney Dis Transplant 2008; 19: $72-5$.

7. Johnston O, O'Kelly P, Donohue J, Walshe JJ, Little DM, Hickey D, et al. Favorable graft survival in renal transplant recipients with polycystic kidney disease. Renal Failure 2005; 27: 309-14.

8. Fitzpatrick PM, Torres VE, Charboneau JW, Offord KP, Holley KE, Zincke H. Long-term outcome of renal transplantation in autosomal polycystic kidney disease. Am J Kidney Dis 1990; 15: 535-43.

9. Niemczyk M, Niemczyk S, Paczek L. Autosomal dominant polycystic kidney disease and transplantation. Ann Transplant 2009; 14: 86-90.

10. Pirson Y, Christophe JL, Goffin E. Outcome of renal replacement therapy in autosomal dominant polycystic kidney disease. Nephrol Dial Transplant 1996; 11 Suppl 6: 24-8.

11. Perrone RD, Ruthazer R, Terrin NC. Survival after endstage renal disease in autosomal dominant polycystic kidney disease: contribution of extrarenal complications to mortality. Am J Kidney Dis 2001; 38: 777-84.

12. Poblete H. XXX Cuenta de hemodiálisis crónica en Chile. Disponible en: http://www.asodi.cl/joomla/archivos/ cuenta\%20XXX\%20HDC\%202010.pdf Consultado el 17 de diciembre de 2011.

13. Hadimeri H, Nordén G, Friman S, Nyberg G. Autosomal dominant polycystic kidney disease in a kidney transplant population. Nephrol Dial Transplant 1997; 12: 1431-6.

14. Brazda E, Ofner D, Riedmann B, Spechtenhauser B, Margreiter R. The effect of nephrectomy on the outcome of renal transplantation in patients with polycystic kidney disease. Ann transplant 1996; 1: 15-8.

15. Bretagnol A, Büchler M, Boutin JM, Nivet H, Lebranchu Y, Chaveau D. Renal transplantation in patients with autosomal dominant polycystic kidney disease: pretransplantation evaluation and follow-up. Nephrol Ther 2007; 3: 449-55.

16. Stiasny B, Ziebell D, Graf S, Hauser IA, Schulze BD. Clinical aspects of renal transplantation in polycystic kidney disease. Clin Nephrol 2002; 58: 16-24.

17. Florijn KW, Chang PC, van der Woude FJ, van Bockel JH, van Saase JL. Long-term cardiovascular morbidity and mortality in autosomal dominant polycystic kidney disease patients after renal transplantation. Transplantation 1994; 57: 73-81.

18. Sanfilippo F, Vaughn W, Peters T, Bollinger R, Spees E. Transplantation for polycystic kidney disease. Transplantation 1983; 36: 54-9. 